\title{
CONECTIVIDAD Y ACCESO A LAS TECNOLOGIAS DE INFORMACIÓN Y COMUNICACIÓN EN LA AMAZONÍA RURAL PERUANA: CASO DE LA CUENCA DEL RÍO NAPO
}

\author{
Luis CALCINA ${ }^{1}$, Bryand HIDALGO ${ }^{2}$ \\ 1 Instituto de Investigaciones de la Amazonía Peruana. Programa de Investigación en Información de la Biodiversidad \\ Amazónica (BIOINF0). Av. José Abelardo Quiñones km 2.5, San Juan Bautista, Maynas - Loreto. luisguet@hotmail.com \\ 2 Ministerio del Ambiente. Especialista en Sistemas de Información Ambiental. Av. Javier Prado Oeste 1440, Lima, Perú.
}

\section{RESUMEN}

Se evaluó la conectividad y el acceso a las tecnologías de información y comunicación (TIC) en diez comunidades de la cuenca del río Napo. El estudio se basó en la aplicación de encuestas, entrevistas y observaciones de campo. Los resultados muestran: a) una lógica expansiva de proyectos tecnológicos que son vistos no como un medio, sino un fin en sí mismos e incursionaron en la Amazonía con perspectivas altamente restrictivas en su alcance y vacías de contenido; b) a su paso, sin proponérselo, generaron una serie de efectos colaterales porque no se tomaron en cuenta las particularidades lingüísticas y culturales de la población. Lo que nos lleva a concluir que lejos de acortar distancias, profundizaron las brechas sociales, no impulsaron un uso adecuado de sus potencialidades como nuevas tecnologías y en vez de democratizar su uso generaron un grupo privilegiado y con exclusividad para acceder a ellas.

PALABRAS CLAVES: Brecha digital, conectividad, Amazonía, TIC, Río Napo.

\section{CONNECTIVITY AND ACCESS TO INFORMATION AND COMMUNICATION TECHNOLOGIES IN RURAL PERUVIAN AMIZON: THE CASE OF THE NAPO RIVER BASIN}

\begin{abstract}
The present work was to evaluate the current state of connectivity and access to Information and Communication Technologies (ICT) in ten communities in the basin of the Napo River. The study was based on the implementation of polls, interviews and field observations. The results show us: a) An expansive logic of technological projects that are seen not as a mean, but as an end and have entered into the Amazonia with highly restrictive prospects in its scope and empty of content; b) On their way, unintentionally, they have generated many collateral effects because they did not have considered the linguistic and cultural characteristics of the local population. This leads us to conclude that far from shorten distances, they have deepened the social gaps and have not promoted a proper use of the ICT potential, instead of democratized its use they have generated a privileged group with exclusivity to access them.
\end{abstract}

KEYWORDS: Digital Gap, Connectivity, Amazonia, ICT, Napo River. 


\section{INTRODUCCIÓN}

Se asume sin mayor discusión que estamos inmersos en la sociedad de la información (SI). Esta postura situacional sobre el mundo nos indica que las actividades humanas están directamente relacionadas con las tecnologías de la información y comunicación (TIC) y presiona para que las colectividades, en su carrera por lograr un alto nivel de desarrollo, deban acceder a las tecnologías a fin de superar la brecha digital.

Bajo estos supuestos la sociedad de la información se entiende como un estadío de desarrollo que permite el intercambio de información de forma instantánea, desde cualquier lugar y en la forma que se prefiera (Oliart, 2009), supone la existencia de una sociedad que se estructura y ordena en función de las TIC y "convierte a la información en un factor de producción, intercambio y consumo" (Giner, 2004) así como en fuente de productividad y poder (Castells, 1999).

Así entendida, su valor más factico nos lleva a sus efectos benéficos en la productividad, pero en su camino van sembrando más dudas que certezas; la información de la que se suele hablar bajo los epígrafes de "era" o "sociedad de la información" no es, dice Arbil (2007) un simple "proceso cognitivo, sino social y cultural y en el más amplio sentido, un proceso a la vez sociotécnico, epistémico y semiótico". Para que la sociedad de la información fuera posible se necesitó que se adopten "determinados medios de producción, intercambio y difusión de conocimiento".

Estos medios y herramientas de producción y distribución del conocimiento se materializan en la conectividad. Por conectividad entenderemos a la capacidad de integrar mediante TIC y redes a diferentes grupos sociales. El nivel de conectividad que se pretende lograr está medido por el grado de penetración de la Banda Ancha (BA). Según el Plan Nacional Para el Desarrollo de la Banda Ancha en el Perú (2011), la BA es entendida como "el acceso a Internet de alta velocidad, que combina la capacidad de conexión (ancho de banda) y la velocidad del tráfico de datos (expresada en bits por segundo bps), permitiendo a los usuarios acceder a diferentes contenidos, aplicaciones y servicios".

La necesidad de incorporar la BA como un aspecto clave en el desarrollo del país nace de la comprobación realizada por una serie de estudios que abonan a favor de esta racionalidad tecnológica. Investigaciones realizadas por el Banco Mundial (2009), "Information and Communications for Development: Extending Reach and Increasing
Impact" demuestra que con un $10 \%$ de aumento de las conexiones de Banda Ancha se incrementa el crecimiento económico de un país en 1,3\%.

Diversas construcciones teóricas y evidencia científicas sustentan el impacto positivo de las TIC en la dinamización económica de las áreas rurales, como es el caso en el uso de celulares en la India (Jensen, 2007), Nigeria (Aker, 2010) o el empleo de cabinas de internet en pueblos en Madhya Pradesh, en la India (Goyal, 2009). En el Perú, estudios realizados en áreas rurales, principalmente de la sierra, dan cuenta que las TIC inciden indirectamente en la productividad, en el incremento de los precios de los productos para los agricultores (Cuanto, 2009), (Barrantes \& Fernández 2012), (Ruiz, 2009), (Fernández \& Medina 2011) e incremento de los salarios y empleabilidad (Katz, 2009).

Todos estos alcances teóricos presentan voluminosos informes que demuestran la incidencia de las TIC en el desarrollo. Informes del Banco Mundial, CEPAL, ECLAC y otros, dan cuenta de esta irrefutable realidad (Peres, 2009). La CEPAL, inclusive, sustenta la multidimensionalidad de las TIC en el desarrollo: aumento de la cobertura en salud, mejora de la calidad de la educación, progreso económico por el acceso de banda ancha, eficiencia de la gestión gubernamental a través del gobierno electrónico y por supuesto moderniza e incrementa el acceso a los mercados.

Surge así un nuevo paradigma basado en "el progresivo aprovechamiento de oportunidades de innovación que puedan medirse de acuerdo con los cambios técnicos fundamentales" (Peres \& Hilbert 2009). Este "paradigma digital" genera a su vez un nuevo "paradigma tecnológico y económico" basado en la digitalización de los procesos que contribuyen al desarrollo económico y social. Paralela a esta línea de argumentación, está la existencia de otro paradigma, el "paradigma económico", donde la información se convierte en mercancía.

El conocimiento se constituye en el factor más importante en la economía moderna asegurando la competitividad de las empresas (Bell, 1990), (Porat, 1977). En la sociedad post moderna -sociedad informacional- señala Lyotard (1991), la información es puesta en circulación según las mismas reglas de la moneda, como pago o inversión. El saber se convierte así en fuerza de producción y en elemento en la competencia por el poder.

Algunos estudiosos plantean muchas dudas sobre la real dimensión de las TIC y en algunos casos son dudas no resultas. Sostiene Tremblay (1996) que un obstáculo epistemológico estriba en el discurso 
sobre las TIC que se caracteriza por un optimismo que, "sin ser desbordante y sin matices, no deja de estar convenido del progresismo" que fortalece la visión unilineal del desarrollo. Es por eso que puede ser un error pretender explicar "el devenir de nuestras sociedades a partir del punto de vista tecnológico y reductor" sobre todo si no se visualizan procesos, culturas e impactos.

Voces más radicales llaman la atención sobre los "nuevos equilibrios" que se han generado a partir de la SI (Burch et al., 2004): 80\% de la producción de información y control sobre las TIC se encuentran en los países centrales, los países periféricos mantienen su condición de subordinación. Las tecnologías en este proceso, se construyen en función de intereses de poder político y económico al nivel mundial, reproduciendo y redimensionando los "desequilibrios" ya existentes (Naim, 2012), (Castells, 1999). "La tecnología no es buena, ni es mala, ni tampoco neutral" sostiene Kransberg (1985).

En este esfuerzo por disminuir la brecha digital e insertarse en la sociedad de la información, en el Perú y en particular en el departamento de Loreto, se pueden observar experiencias en la implementación de proyectos de infraestructura orientados a llevar tecnología e información a las comunidades más alejadas. Explicar el espíritu y el estado actual de estas iniciativas, motiva el presente artículo, que es además un avance de investigación que venimos desarrollando.

La novedad en el tema que nos convoca es que al tratarse de proyectos rurales, muchos de ellos recientemente implementados, han tenido poca repercusión en los centros de investigación o no llamaron aún la atención de los expertos y de formuladores de políticas para el desarrollo. Una vez instalados ya nadie se preocupa de los procesos ex post de estos proyectos o del uso que se hace de estas tecnologías, quizás porque existe la precepción que la tecnología por sí sola solucionará los problemas de la gente y esto no es necesariamente cierto.

Partiendo de los supuestos teóricos y evidencias expuestas, se analizan tres experiencias de implementación de TIC en diez comunidades de la cuenca del río Napo con el objetivo de conocer el estado del arte o estado actual de la conectividad, sus impactos iniciales y posibles alternativas que se pueden sugerir a partir de la situación encontrada.

\section{MATERIALES Y MÉTODOS}

\section{Área de estudio}

El presente estudio fue realizado entre los años 2010 y 2012 en 10 comunidades de la cuenca del Napo (Figura 1), esta cuenca se ubica entre los paralelos $01^{\circ} 00^{\prime} 0$ " y $03^{\circ} 30^{\prime} 0$ " de latitud sur y los meridianos $75^{\circ} 15^{\prime} 00^{\prime \prime}$ y $72^{\circ} 45^{\prime} 0$ " de longitud oeste, con una temperatura promedio anual de $26^{\circ} \mathrm{C}$ y humedad relativa de $86 \%$, con precipitaciones anuales 2000-3000 mm (SENAMHI, 1985).

Geopolíticamente comprende los distritos de Mazán, Napo y Torres Causana, de la provincia de Maynas, departamento de Loreto. Las capitales de distrito Mazán, Santa Clotilde y Cabo Pantoja se encuentran distantes a $120 \mathrm{~km}$ una respecto de la otra y solo son accesibles vía fluvial, son además localidades que se ubican en zona de frontera con Ecuador.

En toda la cuenca se ubican cerca de 33 mil habitantes distribuidos en alrededor de 200 poblados ubicados principalmente en las zonas ribereñas. Esta área de estudio fue seleccionada principalmente porque es un espacio rural amazónico altamente intervenido en materia de tecnologías de la información y comunicación, cuyos impactos o alcances aún no han sido adecuadamente estudiados.

\section{Instrumentos de muestreo y tamaño muestral}

Durante las diferentes etapas del trabajo de campo se realizaron análisis de documentación secundaria, estudios cuantitativos, basado en el método estadístico, aplicando encuestas para medir los niveles de uso, acceso y demanda de información. La muestra fue aleatoria y simple determinándose en 150 las unidades muestrales (con un nivel de confianza de 1,96 y nivel de error deseado de 0,08). Cada unidad muestral corresponde a un hogar.

El cuestionario trabajado estuvo compuesto por 105 preguntas. Su aplicación fue casa en casa tomando en cuenta a un miembro por familia comprendido entre los 15 y 65 años de edad. También se aplicaron estudios cualitativos (etnometodología y etnografía de la comunicación) a través de entrevistas a los actores involucrados así como observaciones de campo que ayudaron a obtener información primaria relevante. 


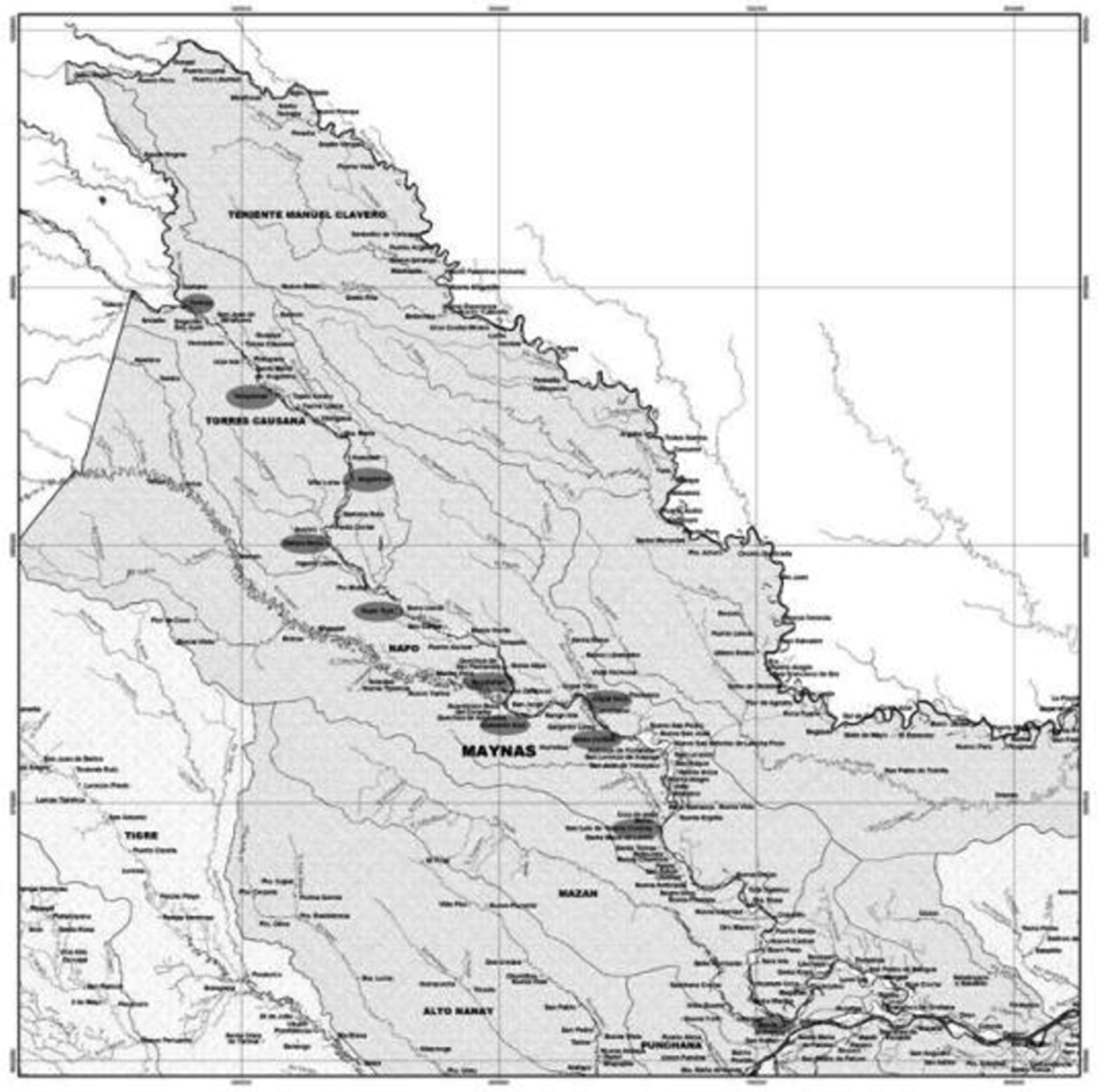

Figura 1: Mapa del departamento de Loreto, indicando la ubicación geográfica de la cuenca del río Napo y las 10 localidades de muestreo consideradas para el estudio.

Fuente: BIOINFO - IIAP (2012) 


\section{RESULTADOS}

\section{Las tic en el napo: una breve descripción}

La cuenca del Napo es una zona considerada entre las más pobres de la región (Tabla 1), a pesar de ello es posible encontrar un alto porcentaje de hogares $(57 \%)$ que cuentan con equipos (televisores, radios, equipos de sonido, lectoras de DVD, alto parlantes) para acceder a contenidos e información. Por otra parte, la cobertura de servicios de telefonía, internet y TV por cable, es moderada presentándose más casos en Mazán por la cercanía a Iquitos. No ocurre lo mismo con el acceso a una computadora y el servicio de Internet.

La infraestructura para el acceso a las TIC se encuentra muy relegada respecto a servicios más convencionales de comunicación. El grueso de respuestas que afirman no usar Internet, tienen varias causas (Tabla 3). No obstante un $85.3 \%$ asegura que si usaría si se logran superar algunas de las dificultades encontradas (accesibilidad y superación de la pobreza digital). El no saber usar la tecnología aparece como una de las razones por la que disminuye el interés de los personas en acceder a las TIC. Destaca también el uso que la población da a las tecnologías y los fines a los que sirve (Tabla 4).

\section{Los esfuerzos por la conectividad en el napo}

En la cuenca del río Napo se identificaron cuatro experiencias principales orientadas a conectar mediante tecnologías de información a la población rural y que fueron implementadas en diferentes momentos.

\section{Telefonía rural}

Desde el año 2000 FITEL implementó proyectos de telefonía fija en las zonas rurales del país y a través de dos procesos de licitación ejecutados (FITEL 2 y 4) se incluye al departamento de Loreto. El servicio usa la red satelital VSAT de Gilat Satellite Network, conocida con el nombre comercial de GILAT (GILAT to home Perú S.A.), que es el actual proveedor del servicio. En la región existen 305 líneas fijas instaladas en Loreto y en mucho menor proporción existen otras redes proveídas por telefónica. Los equipos instalados pueden estar operativos las 24 horas del día gracias al suministro de energía mediante equipos fotovoltaicos (celdas solares).

\section{Telecentros rurales}

Hasta el año 2005 el FITEL instaló veintiséis cabinas en Huánuco, diecisiete en Loreto, cuatro en Madre de Dios, ocho en San Martín y una en Ucayali.
Adicionalmente el Instituto Nacional de Investigación y Capacitación de Telecomunicaciones - Universidad Nacional de Ingeniería (INICTEL - UNI) entre el 2008 y el 2010 implementó una red de telecentros (ocho en total) en las provincias de Maynas (distritos de Las Amazonas, Napo y Mazán) y Requena (distritos de Jenaro Herrera, Emilio San Martín, Puinahua, Sapuena y Tapiche)

Cada una estas "cabinas rurales" tienen seis computadoras personales (laptops), cuentan con servicio de internet y telefonía que utilizan la tecnología VSAT y como fuente de energía emplean paneles solares (sistema fotovoltaico) que alimentan la red en promedio 10 horas al día.

La implementación de los telecentros fue posible gracias a un convenio firmado con los gobiernos locales. De esta manera, INICTEL - UNI aportaba con la infraestructura y asistencia técnica, dejando al municipio la administración y el pago del servicio. En un primer momento estos telecentros como una forma de introducir a la gente en la "era digital" contaban con un programa de fortalecimiento de capacidades en Ofimática Básica con uso de software de código abierto (software libre). Se estima que los telecentros capacitaron alrededor de 60 personas cada uno, con mucho éxito. Culminado este proceso, se realizó la transferencia de los telecentros a los gobiernos locales quienes no supieron darle la importancia necesaria con lo cual perdieron el dinamismo y el impulso inicial.

\section{Proyecto banda ancha satelital (BAS)}

Telefónica del Perú, principal empresa proveedora de servicios de telefonía, televisión por cable e Internet del país, en convenio con el Ministerio de Transportes y Comunicaciones desde al año 2009 vienen implementando el proyecto Banda Ancha para Localidades Alejadas, conocido como BAS. En total han instalado 162 estaciones en Loreto de las cuales una (1) se encuentra instalada en la comunidad Tacsha Curaray, en el distrito del Napo.

El sistema BAS brinda servicios de telefonía pública e Internet de banda ancha fija a tarifas reducidas mediante una plataforma satelital operativa las 24 horas del día. Como en otros casos, la energía se suministra mediante equipos fotovoltaicos (celdas solares) para alimentar baterías que suministran energía al resto de los equipos y a la antena satelital que capta la señal del proveedor.

\section{Red de telemedicina rural}

Desde el año 2009, La Fundación Enlace 
Hispano Americano de Salud (EHAS) y el Grupo de Telecomunicaciones Rurales de la Pontificia Universidad Católica del Perú (GTR-PUCP) impulsaron la implementación de tres redes inalámbricas de voz y datos, de banda ancha, en tres cuencas del departamento de Loreto, Napo, Alto Putumayo y Alto Amazonas (Balsapuerto). Producto de este esfuerzo cerca de 30 puestos y centros de salud han sido interconectados en una extensión que alcanza los $1000 \mathrm{~km}$ de tendido de red.

El caso del Napo se instaló una red WiFi que interconecta a 16 establecimientos de salud con el Hospital Regional de Loreto. Esta red fue diseñada para proveer comunicaciones mediante voz y datos de banda ancha (2 $\mathrm{Mb}$ en promedio). $\mathrm{Su}$ implementación tuvo dos fases; durante el primer período (año 2007) se instaló una primera red de 12 establecimientos entre las localidades Tacsha Curaray y Cabo Pantoja, gracias al "Proyecto de Control de la Malaria en zonas Fronterizas de la Región Andina" PAMAFRO. En la segunda etapa en el marco del Proyecto "Mejora de las condiciones de salud de la población materno - infantil a través del uso apropiado de las Tecnologías de la Información y las Comunicaciones (TIC) en centros y puestos de salud del río Napo (Perú)" se lograron conectar otros cinco establecimientos de salud entre Tacsha Curaray e Iquitos.

La Red del Napo es considerada la Red WiFi más larga del mundo con un recorrido de $450 \mathrm{Km}$. Posee una WiFi de Larga Distancia WiLD (3 Mbds) soportada por una infraestructura de energía de protección y torres muy robusta (de hasta $90 \mathrm{~m}$ ) y con servicios de Telefonía IP, correo electrónico y navegación de Internet. El uso de software libre (libre de licencias) permitió reducir los costos de implementación en todos los proyectos.

A través de esta red es posible hacer un registro y monitoreo epidemiológico de la zona y se convirtió en un gran soporte para el envío y procesamiento de información administrativa. Por otro lado permite hacer consultas por videoconferencia para realizar un diagnóstico más acertado y un tratamiento más adecuado a los pacientes, para ello usa herramientas de apoyo de diagnóstico (estetoscopía, microscopia, ecografía, UCI) para el envío de señales e imágenes médicas a estaciones remotas para su diagnóstico inmediato.

Tabla 1. Distribución muestral y comunidades seleccionadas para la muestra en la cuenca del río Napo

\begin{tabular}{llc}
\hline \multicolumn{1}{c}{ Distrito } & Localidad & Muestra \\
\hline Mazán & Mazán & 23 \\
& Tacsha Curaray & 11 \\
\hline \multirow{2}{*}{ Napo } & Santa Clotilde & 57 \\
& Copal Urco & 8 \\
& Diamante Azul & 10 \\
\hline \multirow{2}{*}{ Torres Causana } & San Rafael & 4 \\
& Campo serio & 14 \\
& Angoteros & 11 \\
\hline & Tempestad & 4 \\
\hline & Cabo Pantoja & 8 \\
\hline
\end{tabular}


Tabla 2. Porcentaje de pobreza monetaria, pobreza extrema de la población y desnutrición en menores de cinco años en la cuenca del río Napo

\begin{tabular}{lcccc}
\hline \multicolumn{1}{c}{ Distrito } & $\begin{array}{c}\text { POBREZA } \\
\text { TOTAL }\end{array}$ & $\begin{array}{c}\text { POBREZA } \\
\text { EXTREMA }\end{array}$ & $\begin{array}{c}\text { DESNUTRICIÓN } \\
\text { CRONICA }\end{array}$ & $\begin{array}{c}\text { DESNUTRICIÓN } \\
\text { AGUDA }\end{array}$ \\
\hline Mazan & 67.1 & 26.7 & 20.4 & 4.2 \\
Napo & 77.1 & 39.7 & 22.7 & 4.0 \\
Torres Causana & 90.6 & 68.4 & 34.4 & 25.0 \\
\hline
\end{tabular}

Fuente: Elaboración propia con datos del INEl 2007 y http://peru.nutrinet.org (2009)

Tabla 3. Razones por las la población de la cuenca del río Napo no usa Internet y tendencias de uso (\%)

\begin{tabular}{llccc}
\hline & & \multicolumn{3}{c}{ Distrito } \\
\cline { 3 - 5 } & & Mazan & Napo & $\begin{array}{c}\text { Torres } \\
\text { Causana }\end{array}$ \\
\hline ¿Explique las razones de por & No necesita & 0,0 & 5,6 & 8,1 \\
qué no usa internet? & No hay & 30,4 & 43,3 & 56,8 \\
& No sabe usar & 43,5 & 30,0 & 27,0 \\
& No tiene dinero & 4,3 & 1,1 & 0,0 \\
& Otra & 21,7 & 20,0 & 8,1 \\
\hline ¿Usaría internet si superan & Si & 95,7 & 84,4 & 81,1 \\
las dificultades? & No & 4,3 & 15,6 & 18,9 \\
\hline & Celular & 26,1 & 2,2 & 2,7 \\
& No usa & 13,0 & 7,8 & 21,6 \\
\hline
\end{tabular}

Fuente: BIOINFO - IIAP 
Tabla 4. Percepción de utilidad de las Tecnologías de Información y Comunicación (TIC) en la población de la cuenca del río Napo (\%)

\begin{tabular}{|c|c|c|c|c|}
\hline & & \multicolumn{3}{|c|}{ Distrito } \\
\hline & & Mazan & Napo & $\begin{array}{l}\text { Torres } \\
\text { Causana }\end{array}$ \\
\hline \multirow{3}{*}{$\begin{array}{l}\text { ¿Considera que el Internet } \\
\text { es útil? }\end{array}$} & $\mathrm{Si}$ & $87,0 \%$ & $81,1 \%$ & $78,4 \%$ \\
\hline & No & $13,0 \%$ & $18,9 \%$ & $13,5 \%$ \\
\hline & No sabe & $0,0 \%$ & $0,0 \%$ & $8,1 \%$ \\
\hline \multirow{9}{*}{$\begin{array}{l}\text { ¿En qué casos lo considera } \\
\text { más útil? }\end{array}$} & Educación/estudios/tareas escolares & $0,0 \%$ & $11,21 \%$ & $8,1 \%$ \\
\hline & Conocer personas & $4,3 \%$ & $0,0 \%$ & $0,0 \%$ \\
\hline & Emergencias familiares/salud & $4,3 \%$ & $22,2 \%$ & $18,9 \%$ \\
\hline & Envío de dinero & $0,0 \%$ & $1,1 \%$ & $0,0 \%$ \\
\hline & Envío de documentos & $0,0 \%$ & $2,2 \%$ & $0,0 \%$ \\
\hline & Comunicación con la familia & $13,0 \%$ & $18,9 \%$ & $21,6 \%$ \\
\hline & Acceso a información/noticias & $60,8 \%$ & $18,9 \%$ & $27,0 \%$ \\
\hline & Negocios & $0,0 \%$ & $0,0 \%$ & $2,7 \%$ \\
\hline & No precisa & $0,0 \%$ & $1,1 \%$ & $0,0 \%$ \\
\hline \multirow{2}{*}{$\begin{array}{l}\text { ¿El teléfono le ayudó a } \\
\text { solucionar algún problema? }\end{array}$} & $\mathrm{Si}$ & $56,5 \%$ & $52,2 \%$ & $62,2 \%$ \\
\hline & No & $43,5 \%$ & $47,8 \%$ & $37,8 \%$ \\
\hline \multirow[t]{12}{*}{ ¿Cómo le ayudó? } & Comunicación: amigos/inmediata/encargos & $52,10 \%$ & $51,10 \%$ & $51,30 \%$ \\
\hline & Comunicarse con familiares & $13,00 \%$ & $11,30 \%$ & $16,30 \%$ \\
\hline & Negocios/compras & $8,70 \%$ & $2,22 \%$ & $5,40 \%$ \\
\hline & Dar servicios & $0,00 \%$ & $1,10 \%$ & $2,70 \%$ \\
\hline & Educación & $0,00 \%$ & $0,00 \%$ & $5,40 \%$ \\
\hline & Emergencias familiares/salud & $4,70 \%$ & $19,90 \%$ & $13,50 \%$ \\
\hline & Comunicar el envío/solicitud de dinero & $0,00 \%$ & $5,50 \%$ & $2,70 \%$ \\
\hline & Comunicar el envío de paquetes & $0,00 \%$ & $2,40 \%$ & $0,00 \%$ \\
\hline & Información de productos/precio de productos & $17,20 \%$ & $4,36 \%$ & $0,00 \%$ \\
\hline & Comunicaciones judiciales & $0,00 \%$ & $2,12 \%$ & $0,00 \%$ \\
\hline & Solicitar servicios & $0,00 \%$ & $0,00 \%$ & $2,70 \%$ \\
\hline & solicitar repuestos & $4,30 \%$ & $0,00 \%$ & $0,00 \%$ \\
\hline
\end{tabular}

Fuente: BIOINFO - IIAP 


\section{DISCUSIÓN}

¿En verdad las tecnologías de información y comunicación están ayudando al crecimiento económico y a mejorar la calidad de vida de esta población? ¿Existe la certeza de que a esa población se les está "conectando" el desarrollo?

La brecha digital no es el problema señala Roberto Bustamante (2011), en un artículo escrito para la revista Tinta Electrónica. En los diagnósticos que realiza, en zonas rurales y urbano-marginales, encuentra que el problema "no es la brecha digital sino la forma como son introducidas las nuevas tecnologías, ya que allí donde han ingresado han ido segregando y discriminando poblaciones". Como veremos, sus percepciones con respecto a los hallazgos en el presente trabajo conducen a resultados similares.

En observaciones realizadas se encuentra que las TIC por sí misma son insuficientes si es que no hay procesos de inducción y capacitación permanentes y sostenidas en el tiempo. En comunidades como Tacsha Curaray dónde se implementó un cabina BAS y es parte de la red de telemedicina rural, las autoridades, líderes locales y productores no usan internet y consideran que éste servicio "solo sirve para el uso de docentes y alumnos". En el caso de los telecentros implementados por el INICTEL - UNI, después de un año capacitar alrededor de 200 personas, dejaron de dictar los cursos de ofimática y nadie ha suplido esta carencia.

Un segundo tema es que las TIC entra en conflicto con saberes locales. Su presencia produce disfunciones comprensivas y refuerza la falta de autoestima. Aquí se da un primer distanciamiento entre los que saben usar la tecnología y los que no saben. El concepto "analfabeto digital" adquiere su real dimensión: acentúa la supuesta "inferioridad" del que no sabe y refuerza la desvaloración de la cultura local. En el marco de los contenidos, desplaza a la información producida localmente por otra que viene de fuera y mejor elaborada. Las comunidades, principalmente indígenas, no están en condiciones de asimilar, procesar ni adaptar estas tecnologías, mucho menos a la velocidad con la que se reproducen porque no disponen de herramientas para redimensionarlas.

El tercer aspecto y a la vez el más importante es que profundizan las diferencias y las distancias. Aquí hablamos de una tecnología exclusiva y excluyente, que no es democratizada y que por la forma como fue planteada genera distancias entre los grupos locales. "A nosotros no nos dejan usar, ellos nomás lo utilizan", señala una señora refiriéndose a los técnicos en salud, que hacen uso de los servicios de la red de Internet.

En visitas a algunas de las estaciones de la red de telemedicina, principalmente en la cuenca del Napo, se comprueba que solo en dos localidades se amplió el uso de telefonía para las escuelas pero en la mayoría de casos la tecnología permanece ausente para la comunidad. La percepción de "distanciamiento" entre la población y la tecnología se hace patente cuando se restringe su uso y alcance.

Como cuarto aspecto, se comprueba que las TIC no necesariamente soluciona los problemas cotidianos de la gente. Cuando se estudia las demandas de la población se encuentra que la gente necesita servicios eléctricos, conexiones de agua y desagüe, apoyo para procesos productivos como piscigranjas, biohuertos $\mathrm{u}$ orientaciones para la siembra y cosecha de sus productos (camu camu, yuca, frejol, plátano, etc.) y las autoridades locales no entienden cómo las TIC les pueden ayudar a atender estas demandas. Además con dos cabinas de internet para 200 centros poblados distribuidos a lo largo de $450 \mathrm{Km}$, lo que se logra es un listado de buenas intenciones antes que soluciones reales.

Como quinto aspecto se tiene que las TIC tienen problemas tecnológicos, no son autónomas, es decir, requieren de otras tecnologías para operar. Muchas cabinas sólo funcionan cuando hay energía eléctrica (generalmente entre las 6:00 p.m. y 9:00 p.m. de la noche) y las que usan paneles solares requieren condiciones atmosféricas favorables. Las fallas de los equipos, si bien no son constantes, ponen en riesgo el funcionamiento de todo el sistema y las torres de soporte de antenas corren el riesgo de colapsar por falta de mantenimiento. El mantenimiento de los equipos se complica por el alto costo del transporte.

Y como sexto y último aspecto se observa que la implementación de las tecnologías resultó insuficiente para atender la demanda. La red de telemedicina del Napo colapsó al tratar de extender su señal a otros servicios. La municipalidad, instituciones del Estado, Policía Nacional, Marina de Guerra, entre otros hacen uso indirectamente de la red afectando su funcionamiento regular.

Como se puede observar, que las tecnologías de la información y comunicación lleguen a las comunidades rurales amazónicas, si bien representan un punto de inflexión entre el todo o la nada, tal como fueron planteadas inducen a su subutilización y restringen su potencialidad. También se puede comprobar que las expectativas teóricas de una tecnología dinamizadora de la economía, capaz de mejorar los precios, incrementar los sueldos o mejorar la empleabilidad, tiene serias restricciones en la realidad rural amazónica. Lo que 
se pretende explicar a partir de esta investigación es llamar la atención sobre el marco teórico del que parten los proyectos TIC y la forma en la que llegan al usuario final: sin valor agregado, sin contenidos y en la mayoría de casos sin alternativas para ampliar su cobertura ni explorar nuevas y mejores utilidades y beneficios.

Estudios realizados por el Banco Interamericano de Desarrollo (BID) señalan que "La evidencia existente está basada en anécdotas que describen casos exitosos, pero que aportan muy poca evidencia científica en cuanto al vínculo entre las TIC y los supuestos aumentos de productividad y bienestar resultantes" (Chong, 2011: 2). Por eso, concluyen que "antes de invertir en la adquisición y ampliación del acceso a las TIC, a los gobiernos les conviene evaluar y fortalecer la capacidad de sus países para usarlas" (Chong, Ibid). Esto debe servir como marco orientador para lograr que la sociedad de la información no sea solo un estadío de desarrollo del mundo, sino una oportunidad para superar las inequidades existentes.

\section{REFERENCIAS BIBLIOGRÁFICAS}

Aker, J; Mbiti, I. 2010. Mobile Phones and Economic Development in Africa. Working Paper. Center for Global Developtment, Washington. $45 \mathrm{pp}$.

Arbil, G. 2007. La información como formación cultural. Cuadernos de Información y Comunicación, 12:59- 73.

BANCO MUNDIAL. 2010 Information and Communications for Development 2009, Extending Reach and Increasing Impact. Banco Mundial, Washington, DC. 340pp.

Barrantes, R.; Fernández, M. 2012. Mobile Phone Use Among Market Traders at Fairs in Rural Peru. USC Annenberg School for Communication y Journalism, 8 (3): 5-52. Disponible en: http://itidjournal.org/itid/article/ view/913. Acceso: 15(05/2012.

Bell, D. 1994. El advenimiento de la sociedad postindustrial. Alianza Editorial, Argentina. 584pp.

Burch, S.; León, O.; Tamayo, E. 2004. Se cayó el sistema. Enredos de la sociedad de la información. Agencia Latinoamericana de Información, Quito. 302pp.

Bustamante, V. 2011. La Brecha Digital no es el problema en el Perú. Tinta Electrónica, 3:21-24. Disponible en: https://es.scribd.com/doc/ 76734406/Brecha-Digital-la-herida-que-sigueabierta-Tinta-Electronica-N-3. Acceso: 20/12/2011.
Castell, M. 1999. La era de la información. Economía, sociedad y cultura. La sociedad Red. Vol. I. Siglo Veintiuno Editores, México. 574pp.

Chong,A.2010. ¿Un mito o un sueño hecho realidad? En A. Chong (editor): Conexiones del desarrollo. Impacto de las nuevas tecnologías de información. BID, Reseña. 40pp.

Fernández, R.; Medina, P. 2011. Evaluación del impacto de acceso a las TIC sobre el ingreso de los hogares. Una aproximación a partir de la metodología Propensity Score Matching y datos de panel para el caso peruano. Diálogo Regional sobre Sociedad de la Información, DIRSI, Lima. $76 \mathrm{p}$.

Giner, F. 2004. Los sistemas de información en la sociedad del conocimiento. ESIC Editorial, Madrid. 216pp.

GOBIERNO DEL PERU. 2012 Plan Nacional para el Desarrollo de la Banda Ancha en el Perú, Lima. 241pp.

Goyal, A. 2010. Information, Direct Access to Farmers, and Rural Market Performance in Central India. American Econmic Journal: Applied Economics, 2: 22-45.

INSTITUTO CUANTO. 2009. Selección de una empresa consultora que se encargue de realizar el estudio de evaluación de impacto de proyectos de FITEL. Informe Final, Tomo I, Lima. 437pp.

INSTITUTO NACIONAL DE ESTADÍSTICA E INFORMÁTICA. 2008. Censos Nacionales 2007: XI de Población y VI de Vivienda [multimedia] Base Redatam.

Jensen, R. 2007. The Digital Provide: Information (Technology), market performance and Welfare in the South Indian Fisheries Sector. Quarterly Journal of Economics, 122 (3): 879-925. Disponible en: ttp://qje.oxfordjournals.org/ content/122/3/879.full.pdf + htm. Acceso: 20/06/2011.

Katz, R. 2009. El papel de las TIC en el desarrollo. Propuestas de América Latina a los retos económicos actuales. Ediciones Ariel, Colección Fundación Telefónica, España,192pp.

Kranzberg, M.; Pursell, C. Jr. (ed.). 1976. Technology in Western Civilization. Nova York: Oxford University Press, 2 vol.

Lyotar, F. \& Rato. M. (tradiccion).1991. La condición post moderna. Informe sobre el saber. Editorial Catedra, España. 120 pp.

Naim, M. 2012. Un Internet para los ricos y otra para los demás. Diario El País de España, 29 de abril de 2012. Disponible en (http://internacional. elpais.com/internacional/2012/04/29/actualidad 
/1335676157529701.htm). Acceso: $10 / 05 / 2012$

Oliart, B. (Edición). 2002. La Sociedad de la Información en el Perú. Presente y perspectivas 2003-2005. Servicios Editoriales del Perú, Telefónica del Perú, Lima. 100pp.

Peres, W.; Hilbert, M. (Editores). 2009. La sociedad de la información en América Latina y el Caribe. Desarrollo de las tecnologías para el desarrollo. CEPAL, Chile. 16pp.

Porat, M. 1977. The Information Economy: Definition and Measurement. United Satetes Department of Comerce, Washington. 319pp.

Ruiz, L. 2009. Impacto de la telefonía celular en zonas rurales pobres del Perú. Proceeding $p f$ the 3erd ACORN-REDECOM Conference Mexico City. México. 12pp.

Siquiera B.; César R. 2010. Sociedad de la Información: reestructuración capitalista y esfera pública global. IC-Revista Científica de Información y Comunicación, 7: 35 - 58. Disponible en: https://ipena44.files.wordpress. com/2013/02/1292341785-12siqueirabaja.pdf. Acceso: 15/05/2012.

Tremblay, Gaëtan. 1996. La societat de la informació: del fordisme al gatesisme. Revista Análisis, 19: 57-78.

Recibido: 22/05/2014

Aceptado para publicación: 23/07/2014 
